\title{
A simple estimation method to find the proper capacity of a combined heat and power unit
}

\author{
Woojin Cho ${ }^{1}$, Janghyun Kim ${ }^{1}$, Kwan-Soo Lee ${ }^{1, *}$, Seung-Kil Son ${ }^{2}$, Kwon Woo Lee ${ }^{2}$ \\ ${ }^{I}$ School of mechanical engineering, Hanyang University, Seoul, Republic of Korea \\ ${ }^{2} R \& D$ center, KD Navien co., ltd., 459-2 Gasan-dong, Geumcheon-gu, Seoul 153-803, Republic of Korea \\ *Corresponding author.Tel: +82-2-2220-0426, Fax:+82-2-2295-9021,E-mail: ksleehy@hanyang.ac.kr
}

\begin{abstract}
Selecting a proper capacity of the CHP unit is a complicated problem where energy usage pattern of end users and energy price structures must be considered. Thus, advanced computer simulations are used to predict the proper capacity of the unit, however, certain methods require expensive information such as part load performance of the unit and load profiles of end users. This paper suggests a simple method of predicting proper capacity by presenting analytical equations which calculate annual savings with different capacity of the CHP unit. These equations have merit which can be applied to various conditions by covering the energy usage patterns and energy price structures.
\end{abstract}

Keywords: Combined heat and power, Capacity

\section{Nomenclature}

$c_{e} \quad$ price of electricity........................ents $/ \mathrm{kWh}$

$c_{f} \quad$ price of fuel..................................ents $/ \mathrm{kWh}$

$c_{t} \quad$ price of heat .................................cents $/ \mathrm{kWh}$

hpr heat to power ratio ........................ $\mathrm{kW} / \mathrm{kW}$

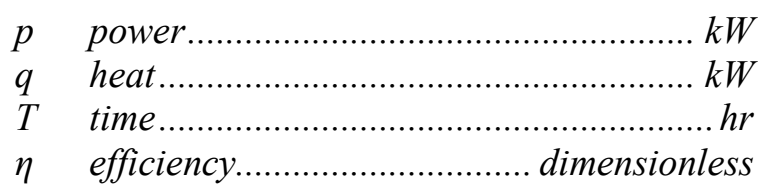

\section{Introduction}

Although a combined heat and power (CHP) unit produces both heat and electricity, it cannot control the ratio of output heat to power (heat-to-power ratio). Thus there is a potential discrepancy between the heat/electricity production of a CHP unit and the heat/electricity consumption on the demand side. This issue can be resolved by using a power grid and supplementary water heating system in conjunction with the CHP unit. However, the varying prices of energy consumed by the CHP unit, power grid, and supplementary heating system complicate the proper choice of CHP unit size. This problem is significantly different from selecting a boiler size solely on the basis of the consumption of heat by the end user.

Contrary to expectation, there are only few existing studies on selecting the optimum capacity of a CHP unit. It is certain that appropriate criteria for selecting the proper capacity of a CHP unit cannot be derived from a case study based on parameters that are limited, or classified only into 3 or 4 levels. Procedures such as modeling techniques, mathematical optimization, or simulation programs obtained from other researchers could help to provide relevant standards. However, these methods are actually difficult to adopt, and the use of other people's results requires expensive information, such as long-term accumulated minute-byminute load profiles. The performance of a CHP unit varies according to the manufacturer, and energy usage patterns vary from one household to the next. Moreover, energy prices related to the use of a CHP system vary in different regions. Thus, although a solution to the problem of selecting the proper capacity of a CHP unit should account for parameters such as system performance, pattern of energy usage, and energy price structure, the required methodology is not easily found in the literature. 
This study uses information that is easily obtained (annual average heat load, base/peak power load), and presents a simple and intuitive method for predicting proper CHP unit capacity for

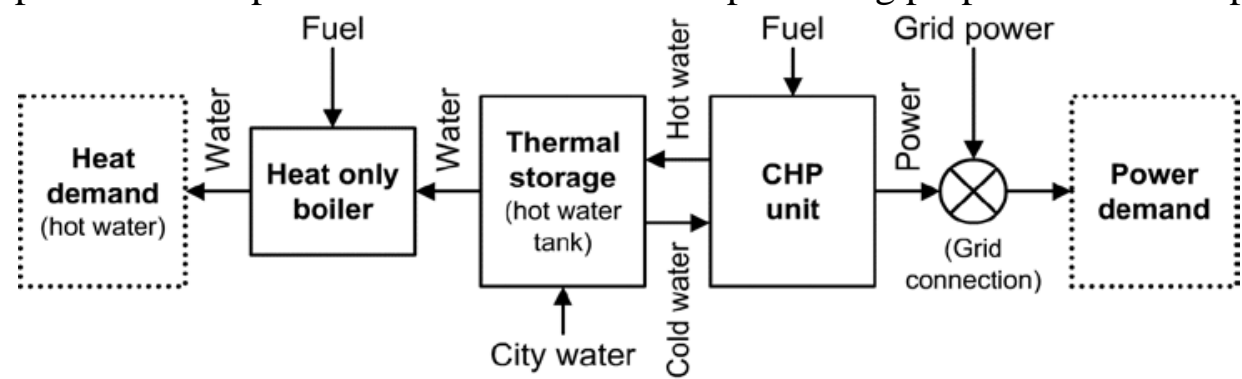

Figure 1. Schematic of the CHP system

end users. The final result is a set of equations for calculating the proper capacity for three classifications (three types of energy usage patterns), and is verified via computer simulation.

\section{CHP system}

This research is based on a CHP system that includes a CHP unit, thermal storage unit, and HOB (heat only boiler), as shown in Figure 1. The CHP unit operates when there is a heat demand, and the power output of the unit follows the power load on the demand side. This operating strategy is called the "following heat demand and chasing power load" (FHD-CPL) strategy, and is usually preferred where selling electricity back to the grid is impossible, or offers no economic advantage.

\section{Problem formulation}

In this paper, the objective function for determining the proper capacity is defined as the annual economic savings from using the CHP system. The problem is formulated as follows. The cost of energy consumption per unit time step by a CHP system is denoted by $e c_{C H P}$, and the cost of energy consumption per unit time step by a separated heat and power (SHP) system is denoted by $e c_{S H P}$. Equation (1) presents the formulation procedure and its result. When an arbitrary time $T$ is divided into $N$ intervals of length $h$, the cost saving in the $\mathrm{n}^{\text {th }}$ interval is calculated as follows:

$$
\begin{gathered}
E C R_{n}=h\left(e c_{C H P, n}-e c_{S H P, n}\right)=h \cdot c_{t} \cdot q_{T S, n}+h \cdot \delta_{n} \cdot f_{n} \\
f_{n}=\left(c_{e}-c_{e} \max \left(1-r_{n}, 0\right)-\left(c_{f} / \eta_{e, n}\right) r_{n}\right) p_{\text {load }, n}
\end{gathered}
$$

where $r_{n}$ is the power share of the CHP unit against the power load, $\eta_{e}$ is the electrical efficiency of the unit, and $c_{t}$ is the equivalent price of the energy. The above equations are also represented in Figure 2.

$$
r_{n}=p_{C H P, n} / p_{l o a d, n}, \quad \eta_{e, n}=p_{C H P, n} / f_{C H P, n}, \quad c_{t}=c_{t}^{*} / \eta_{t, H O B}
$$

Also, $\delta_{n}$ is a parameter which value is 0 or 1 depending on the CHP units operation status in $\mathrm{n}^{\text {th }}$ interval.

$$
\delta_{n}= \begin{cases}1 & \text { if CHP unit runs at } n \\ 0 & \text { if CHP unit halts at } n\end{cases}
$$




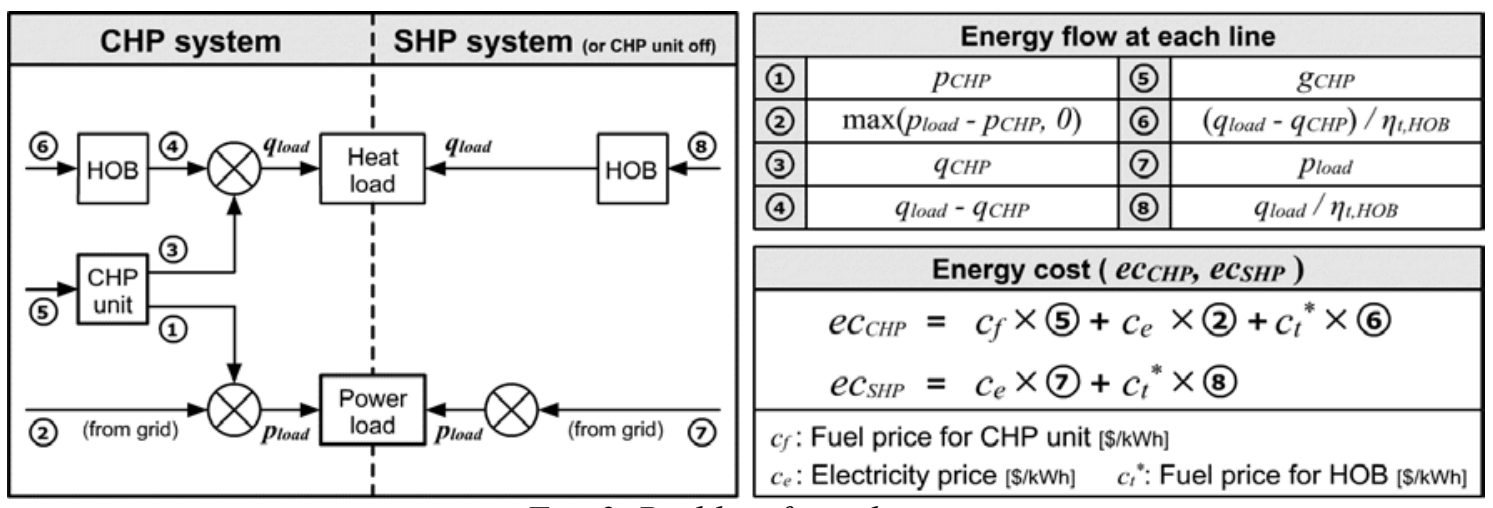

Fig. 2. Problem formulation

The total $E C R$ (economic cost reduction) over an arbitrary period $T$ is the sum of the $E C R_{n}$ of the individual intervals.

$$
E C R=\sum_{n=1}^{N} E C R_{n}=h \sum_{n=1}^{N}\left(c_{t} \cdot q_{T S, n}\right)+h \sum_{n=1}^{N} f_{n}
$$

The first term on the right-hand side of Equation (5) represents the total amount of heat supplied to the end user over period $T$. Since only heat from the CHP unit is supplied to the thermal storage unit, this amount is the same as the total thermal output of the CHP unit over the period.

$$
Q_{T S} \square h \sum_{n=1}^{N}\left(\delta_{n} \cdot q_{C H P, n}\right)=h \sum_{n=1}^{N}\left(\delta_{n} \cdot h p r_{n} \cdot r_{n} \cdot p_{\text {load }, n}\right)
$$

In the above equation, $h p r_{n}$ denotes the ratio of the electrical output to the thermal output.

$$
h p r_{n}=q_{C H P, n} / p_{C H P, n}
$$

Equations (5), (6) and (7) can be rearranged as follows:

$$
\begin{gathered}
E C R=h \sum_{n=1}^{N}\left(\delta_{n} \cdot e c r_{n} \cdot p_{\text {load }, n}\right) \\
e c r_{n}=c_{t} \cdot h p r_{n}+c_{e} \min \left(r_{n}, 1\right)-\left(c_{f} / \eta_{e, n}\right) r_{n}
\end{gathered}
$$

The maximum power load during period $T$ is denoted by $p_{\text {peak }}$, and the range from 0 to $p_{\text {peak }}$ is divided into a uniform number of classes $M$. The $\mathrm{m}^{\text {th }}$ class frequency, corresponding to the class mark $p_{\text {load }, m}$, can be expressed as a function $\mathrm{n}\left(p_{\text {load,m }}\right)$, and the mathematical relationship between the period $T$, the number of intervals $N$, the number of classes $M$, and the frequency of each class $\mathrm{n}\left(p_{\text {load }, m}\right)$ is given by

$$
\begin{gathered}
T=h \cdot N=h \sum_{m=1}^{M} \mathrm{n}\left(p_{\text {load }, m}\right) \\
\sum_{n=1}^{N} p_{\text {load }, n}=\sum_{m=1}^{M}\left\{p_{\text {load }, m} \cdot \mathrm{n}\left(p_{\text {load }, m}\right)\right\}
\end{gathered}
$$


The following equation for ECR can be obtained by rearranging Equations (8), (10) and (11):

$$
E C R=T \sum_{m=1}^{M}\left\{o r_{m} \cdot e c r_{m} \cdot p_{\text {load }, m} \cdot \mathrm{n}\left(p_{\text {load }, m}\right) / N\right\}
$$

where $o r_{m}$ is the availability factor of the CHP unit in the $\mathrm{m}^{\text {th }}$ class. Over period $T$, $o r_{m}$ is assumed to have a constant value or, which is unrelated to any of the classes.

$$
o r_{m}=\left(\sum \delta_{n} \mid p_{\text {load }, n} \in p_{\text {load }, m}\right) / \mathrm{n}\left(p_{\text {load }, m}\right) \approx \text { or }
$$

For an infinitely large number of intervals $N$ and number of classes $M$, Equation (12) can be expressed as follows:

$$
\begin{gathered}
E C R=T \cdot \text { or } \cdot \overline{e c r} \\
\overline{e c r}=\int_{0}^{p_{\text {peak }}} \operatorname{ecr}\left(p_{\text {load }, m}, p_{C H P, \text { nom }}\right) \cdot p_{\text {load }, m} \cdot \operatorname{pdf}\left(p_{\text {load }}\right) d p_{\text {load }}
\end{gathered}
$$

Here $\operatorname{PDF}\left(p_{\text {load }}\right)$ is the probability density function of the continuous random variable $p_{\text {load }}$.

To satisfy Equation (6), the heat and power load over period $T$ should be periodic. Also, the thermal storage unit should compensate for any discrepancies between thermal output and heat load over period $T$. Thus it is appropriate to define period $T$ to be a day. The annual energy cost savings are then calculated as follows:

$$
A E C R=\sum_{\text {day }=1}^{365} E C R_{\text {day }}=\sum_{\text {day }=1}^{365}\left\{T_{\text {day }} \cdot o r_{\text {day }} \cdot \overline{e c r_{d a y}}\right\}
$$

Also, for the demand side (which has a similar annual power load distribution pattern), Equation (16) can be simplified as follows:

$$
\begin{gathered}
A E C R=T_{\text {year }} \cdot \overline{o r_{\text {year }}} \cdot \overline{e c r_{\text {year }}} \\
\overline{\text { or }} \text { year } \\
=\sum_{\text {day }=1}^{365} o r_{\text {day }} / 365
\end{gathered}
$$

\section{Simple estimation via linearization}

In this paper, a simple estimation method for predicting the proper capacity of a CHP unit is proposed, based on linearization of the non-linear functions in Equation (17).

\subsection{Partial load performance of the CHP unit and CDF of the power load}

Equation (9) includes $\eta_{e}$ and $h p r$, which are related to the performance of the CHP unit. In this study, the partial load performance is assumed to be as shown in Figure 3 (a). Applying this to Equation (9), and substituting into Equation (15) yields

$$
\overline{e c r}=\left\{\alpha+\beta \cdot \operatorname{cdf}\left(p_{C H P, \text { nom }}\right)\right\} p_{C H P, \text { nom }}
$$




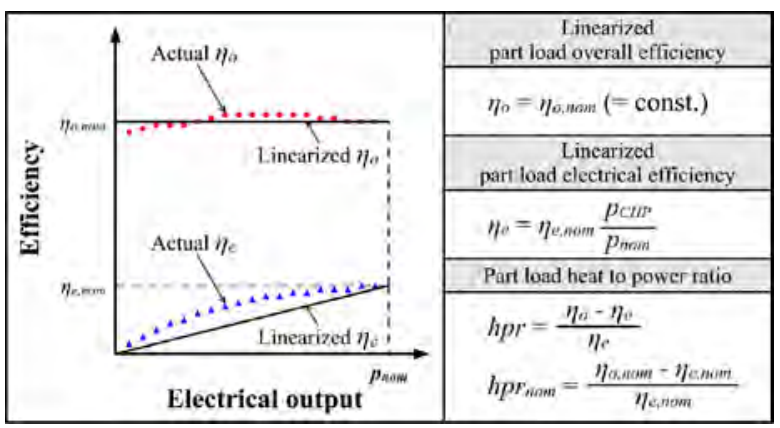

(a) Partial load linearization

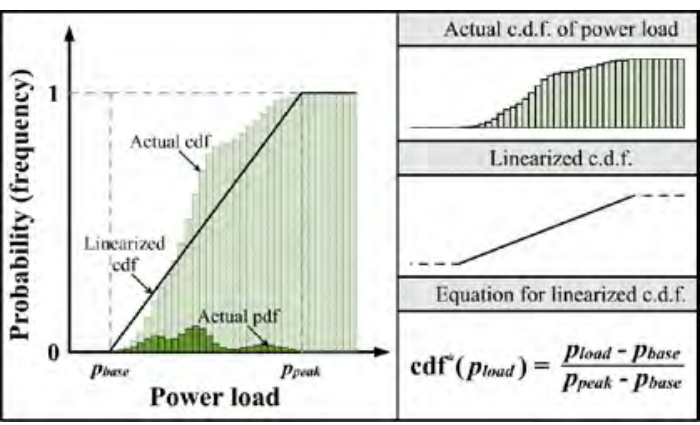

(b) CDF of power load

Figure 3. Part load performance and cumulative distribution function

Here,

$$
\alpha=c_{t} \cdot h p r_{\text {nom }}+c_{e}-c_{f} / \eta_{e, \text { nom }}, \quad \beta=c_{t}-c_{e}, \quad \operatorname{cdf}\left(p_{\text {load }}\right)=\int_{0}^{p_{\text {load }}} \operatorname{pdf}(p) d p
$$

The PDF and cumulative distribution function (CDF) of the demand-side power load follow the pattern shown in Figure 3 (b). The CDF in Equations (20) is assumed to be a first-order function, as shown in Figure 3 (b). The assumed CDF for each interval, based on division in terms of the base power load and peak power load of the demand side, is as follows:

$$
\operatorname{cdf}^{*}(p)= \begin{cases}0 & \left(p<p_{\text {base }}\right) \\ \left(p-p_{\text {base }}\right) /\left(p_{\text {peak }}-p_{\text {base }}\right) & \left(p_{\text {base }} \leq p<p_{\text {peak }}\right) \\ 1 & \left(p \geq p_{\text {peak }}\right)\end{cases}
$$

\subsection{Distribution of the daily average heat load}

The maximum daily average heat load of the demand side is denoted by $\overline{q_{\max }}$, and the minimum daily average heat load of the demand side is denoted by $\overline{q_{m \iota n}}$. If the daily average heat load for the $\mathrm{k}^{\text {th }}$ day is assumed to be a first-order function, as shown in Figure 4 (a), or year in Equation (18) can be obtained as shown in Figure 4 (b). Based on the procedure illustrated in Figure 4 (b), or $_{\text {year }}$ can be expressed as a function of $p_{\text {nom }}$ as follows:

$$
\overline{\text { or }_{\text {year }}} \square \begin{cases}1 & \text { for } p_{\text {nom }}<\overline{q_{\text {load }}} /\left\{h p r+\operatorname{cdf}\left(p_{\text {nom }}\right)\right\} \\ \overline{q_{\text {load }}} /\left[\left\{h p r+\operatorname{cdf}\left(p_{\text {nom }}\right)\right\} \cdot p_{\text {nom }}\right] & \text { for } \quad p_{\text {nom }} \geq \overline{q_{\text {load }}} /\left\{h p r+\operatorname{cdf}\left(p_{\text {nom }}\right)\right\}\end{cases}
$$

Here,

$$
\overline{q_{\text {laod }}}=\left(\overline{q_{\max }}+\overline{q_{\min }}\right) / 2
$$

Thus or can be calculated solely on the basis of the CDF of the average annual heat $\left(q_{\text {load }}\right)$ and the power load on the demand side. 


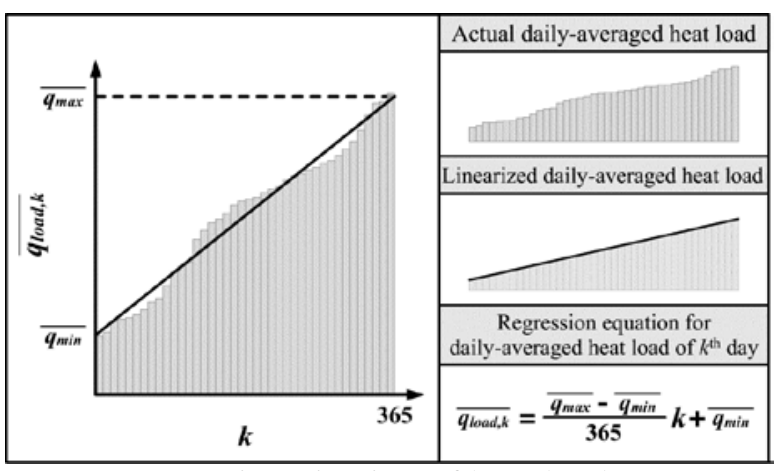

(a) Linearization of heat load

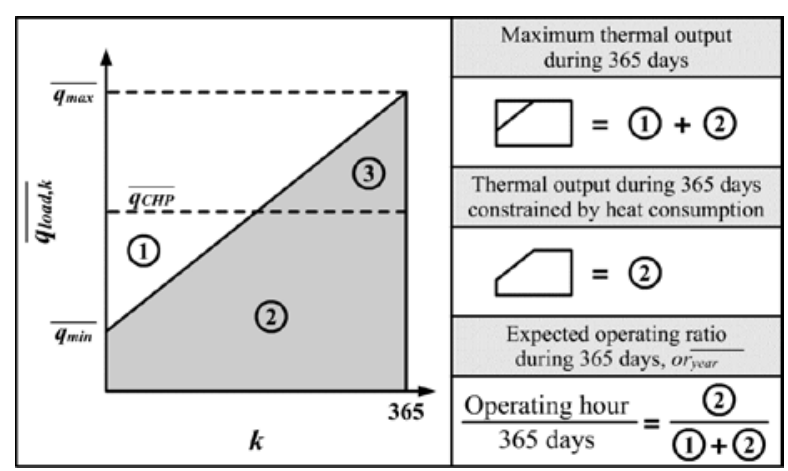

(b) Calculation of operating ratio

Figure 4. Distribution of daily-averaged heat load

\section{Simple estimation method}

Equation (17) can be written as follows:

$$
A E C R=T_{\text {year }} \underbrace{\left(\overline{\text { or }_{\text {year }}} \cdot p_{\text {nom }}\right)}_{\mathrm{U}} \underbrace{\left(\overline{e c r_{\text {year }}} / p_{\text {nom }}\right)}_{\mathrm{V}}
$$

In Figure 5, $p_{\text {nom }}$ is divided into intervals according to energy usage patterns (Type 1, Type 2, Type 3), and the forms of $U$ and $V$ in Equation (24) are graphically expressed for each interval. The proper capacity $p_{\text {nom,pr }}$ can be obtained by finding the maximum value of this simple polynomial (the order of UV is less than 2 over the entire range). The proper capacities for each type are shown in Table 1, based on the simple estimation method. However, one should be aware that this method does not provide the optimal capacities when $\alpha$ is negative or $b$ is positive. In these circumstances, a CHP system cannot guarantee an economic advantage.

\section{Validation}

We verified the performance of our technique with a previously validated simulation program. This program was developed in earlier research, and the results (such as the amount of electricity generated and the thermal output) were obtained from a simulation based on the end user's load profiles. The details of this program are not included in the present paper, but can be found in the article "Optimum generation capacities of micro combined heat and

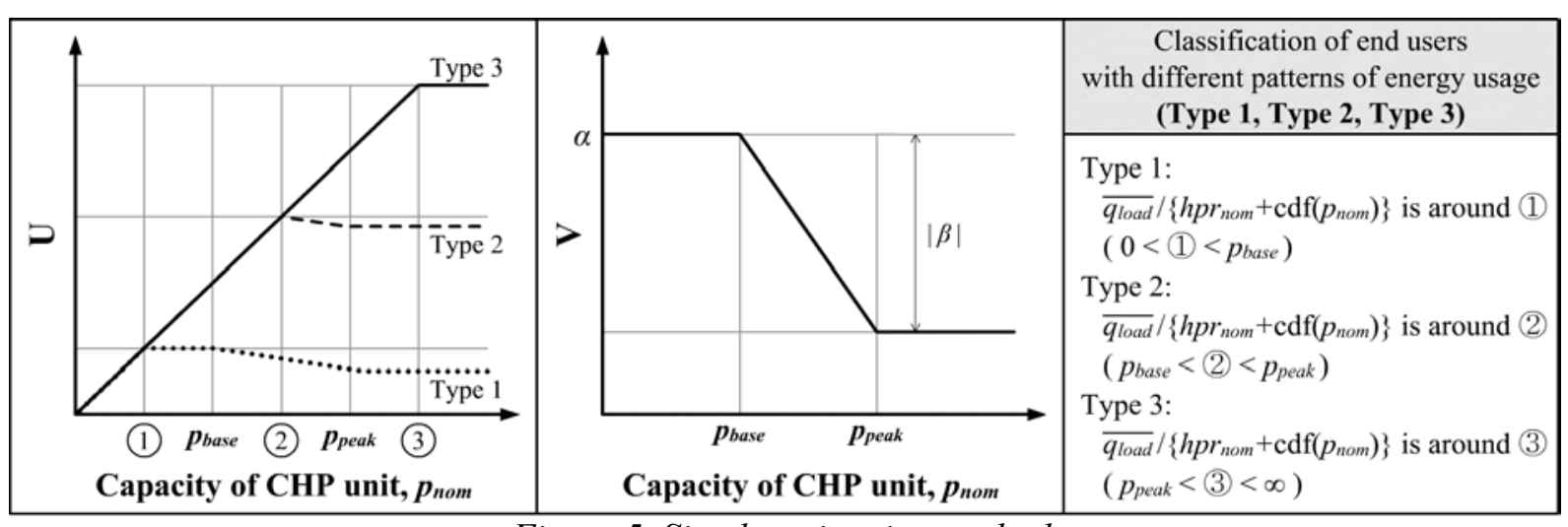

Figure 5. Simple estimation method 
Table 1. Proper capacities for each type

\begin{tabular}{|c|c|}
\hline $\begin{array}{c}\text { Pattern } \\
\text { of energy usage }\end{array}$ & Proper capacity \\
\hline Type 1 & $p_{\text {nom }, p r}=\overline{q_{\text {load }}} / h p r_{\text {nom }}$ \\
\hline Type 2 & $\begin{array}{l}\alpha / \beta \geq-p_{\text {base }} /\left(p_{\text {peak }}-p_{\text {base }}\right) \rightarrow p_{\text {nom }, p r}=p_{\text {base }} \\
\alpha / \beta<\left(p_{\text {base }}-2 \overline{q_{\text {load }}} / \overline{h p r}\right) /\left(p_{\text {peak }}-p_{\text {base }}\right) \rightarrow p_{\text {nom }, p r}=\overline{q_{\text {load }}} / \overline{h p r} \\
\text { otherwise } \rightarrow p_{\text {nom }, p r}=\left\{(1-\alpha / \beta) p_{\text {base }}+(\alpha / \beta) p_{\text {base }}\right\} / 2\end{array}$ \\
\hline Type 3 & $\begin{array}{l}\alpha / \beta \geq-p_{\text {base }} /\left(p_{\text {peak }}-p_{\text {base }}\right) \rightarrow p_{\text {nom }, p r}=p_{\text {base }} \\
\alpha / \beta<\left(p_{\text {base }}-2 p_{\text {peak }}\right) /\left(p_{\text {peak }}-p_{\text {base }}\right) \rightarrow p_{\text {nom }, p r}=\overline{q_{\text {load }}} / \overline{h p r} \\
\text { otherwise } \rightarrow p_{\text {nom, }, p r}=\left\{(1-\alpha / \beta) p_{\text {base }}+(\alpha / \beta) p_{\text {base }}\right\} / 2\end{array}$ \\
\hline Parameters & $\begin{array}{l}\alpha=c_{t} \cdot h p r_{\text {nom }}+c_{e}-\frac{c_{f}}{\eta_{e, n o m}}, \quad \beta=c_{t}-c_{e}, \quad \overline{h p r}=h p r_{\text {nom }}+X \\
X=-A+\left\{A^{2}+\frac{\overline{q_{\text {load }}}-h p r_{\text {nom }} \cdot p_{\text {base }}}{p_{\text {peak }}-p_{\text {base }}}\right\}^{1 / 2}, \quad A=\frac{1}{2}\left\{h p r_{\text {nom }}+\frac{p_{\text {base }}}{p_{\text {peak }}-p_{\text {base }}}\right\}\end{array}$ \\
\hline
\end{tabular}

power systems in apartment complexes with varying numbers of apartment units."[1]

The AECR (annual economic cost reduction) result derived from Equation (24) was compared with that obtained from the simulation (numerical experiment). The partial load performance, demand-side energy usage pattern, and other information used in each method are listed in Table 2. We point out that the predictions obtained by the simple estimation method were based solely on the boldface entries in Table 2 .

Table 2. Information used in each method

\begin{tabular}{|c|c|c|}
\hline & Simulation program & $\begin{array}{c}\text { Simple } \\
\text { estimation }\end{array}$ \\
\hline$\eta_{e, n o m}, h p r_{n o m}$ & $0.32,1.78[1]$ & $0.32,1.78$ \\
\hline$\eta_{e}(l f) / \eta_{e, n o m}$ & $1.03-0.98 \cdot 0.0315^{l f}[1]$ & lf \\
\hline $\operatorname{hpr}(l f) / h p r_{n o m}$ & $\left(0.96+0.76 \cdot 0.0454^{l f}\right) /\left(1.03-0.98 \cdot 0.0315^{l f}\right)[1]$ & 1 \\
\hline $\begin{array}{l}\text { Distribution of } p_{\text {load }} \\
\text { (PDF and CDF) }\end{array}$ & (Simulation uses load profiles [1]) & $\begin{array}{c}\text { Using equation }(21) \\
\left(p_{\text {base }}=\mathbf{1 0} \mathbf{~ k W}, p_{\text {peak }}=\mathbf{2 8 0} \mathbf{k W}\right)\end{array}$ \\
\hline $\begin{array}{c}\text { Annual-averaged } \\
\text { heat load }\end{array}$ & (Simulation uses load profile [1]) & $242 \mathrm{~kW}$ \\
\hline$c_{t}, c_{e}, c_{f}$ & \multicolumn{2}{|c|}{6 cents/kWh, 12 cents/kWh, 6 cents/kWh (both) } \\
\hline
\end{tabular}




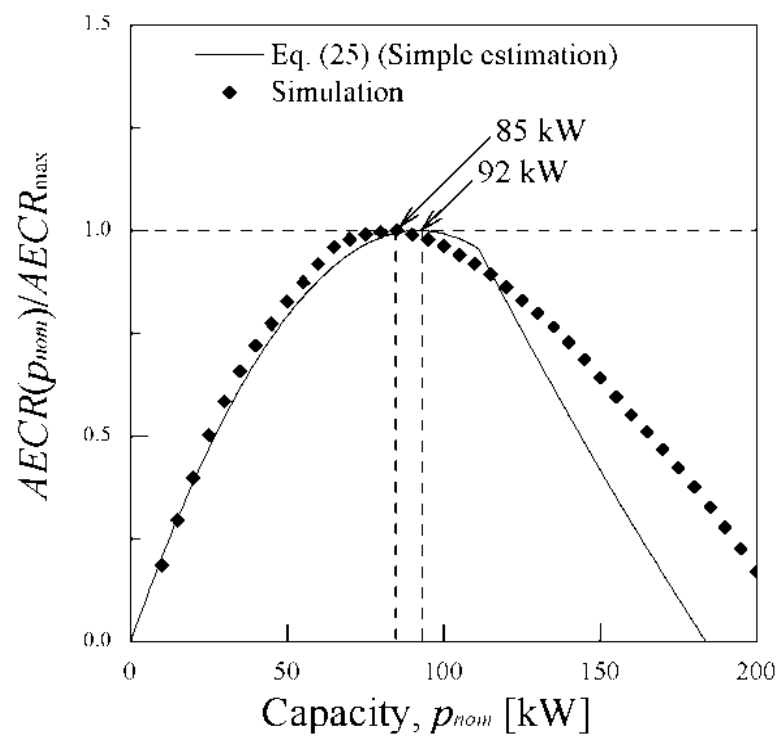

Figure 6. Comparison of the simple estimation method and a previous study

Figure 6 compares the standardized AECRs of each method (AECRs of different capacities, $\operatorname{AECR}\left(p_{\text {nom }}\right)$ divided by the maximum AECR, $\left.A E C R_{\max }\right)$. The AECR calculated via Equation (24) attained a maximum at $p_{\text {nom }}=92 \mathrm{~kW}$, while the AECR calculated via the simulation program reached a maximum at $p_{\text {nom }}=85 \mathrm{~kW}$. Hence the results were in good agreement. In this manner, we can calculate the maximum (proper capacity) of a CHP unit by using the simple estimation method shown in Table 1.

\section{Conclusions}

In this paper, a simple estimation method was proposed to predict the proper capacity of a CHP unit. The advantages of this technique are described below.

First, if the relevant information (such as electrical and thermal load profiles and nominal efficiencies of the CHP system) are opened to the public, the technique proposed in this paper provides useful method to estimate the proper capacity of the micro-CHP system without using complicated (and expensive) simulation programs suggested from others.

Second, the method that has been suggested in this paper could handle different kinds of conditions by employing various energy prices and system's performances. For example, it could be applied into different situations in various countries (having different energy price policies and different CHP prime movers such as stirling or turbine engine).

Third, the most important aspect in this paper is that we suggested the simplest model among the others which can be cost-effective and easy to apply.

\section{Reference}

[1] J. Kim, W. Cho, K.-S. Lee, Optimum generation capacities of micro combined heat and power systems in apartment complexes with varying numbers of apartment units, Energy, 2010, Vol.35, Issue 12, pp. 5121-5131. 